\title{
Higgs Decay into Bottom Quarks Seen at Last
}

\author{
Two CERN experiments have observed the most probable decay channel of the Higgs \\ boson-a milestone in the pursuit to confirm whether this remarkable particle behaves as \\ physicists expect.
}

\section{by Howard E. Haber*}

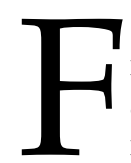
ifty years ago, Steven Weinberg and Abdus Salam independently proposed a theory for the weak interactions that govern certain nuclear processes such as radioactive beta decay [1]. The particles that mediate these interactions, the $W$ and $Z$ bosons, had to be massive to explain the short-range nature of the weak nuclear force. But in order to introduce these masses without otherwise

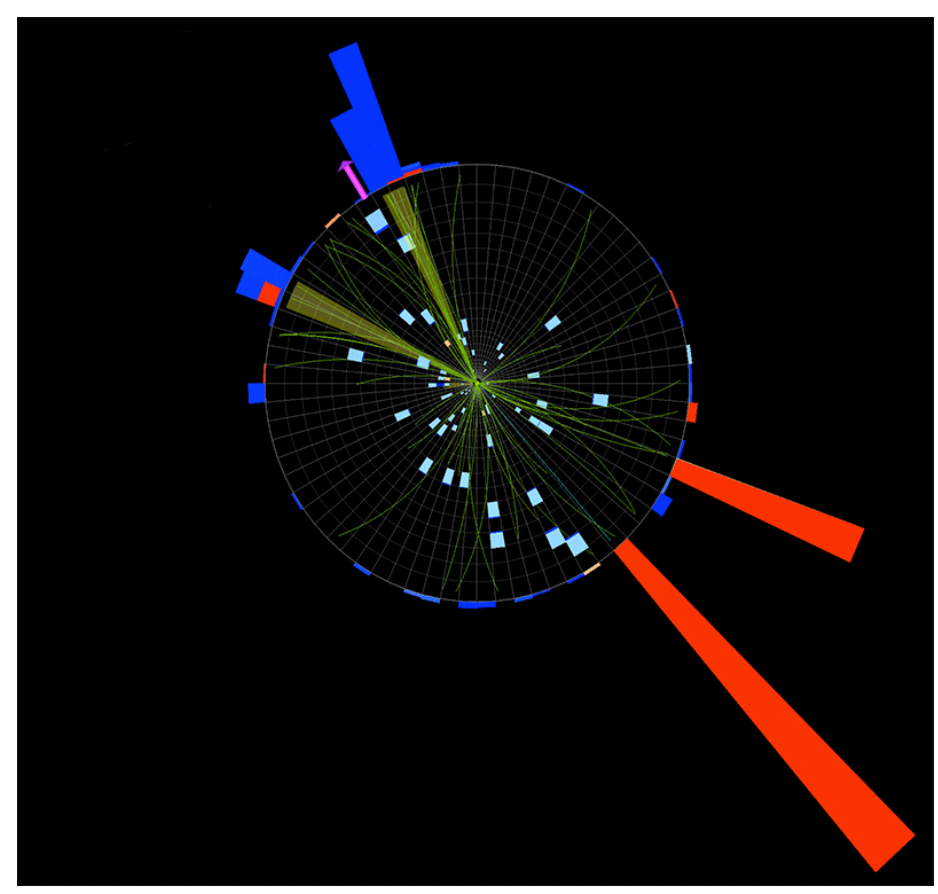

Figure 1: The ATLAS and CMS collaborations at CERN have observed the decay of the Higgs boson into a pair of bottom quarks, the particle's most probable decay channel. (CMS Collaboration)

\footnotetext{
* Santa Cruz Institute for Particle Physics, University of California,
} Santa Cruz, CA, USA destroying the mathematical consistency of the theory, Weinberg and Salam assumed that the $W$ and $Z$ bosons acquire mass by interacting with an omnipresent field-an idea that Peter Higgs and a number of other theorists had proposed earlier $[2,3]$. The presence of a "Higgs field" implied the existence of a new particle [3], the Higgs boson, which, after decades of searching, was ultimately discovered in 2012 in the debris of proton-proton collisions by the ATLAS and CMS collaborations at the Large Hadron Collider (LHC) at CERN [4].

The 2012 discovery was a triumph for particle physics, but it was also the beginning of a new pursuit: determining whether physicists have the right picture of how the Higgs boson interacts with other particles. These interactions make the Higgs boson highly unstable, causing it to decay into a number of different possible final states. The CMS and ATLAS collaborations have now confirmed a central part of the current picture by observing the decay of the Higgs boson into a pair of bottom quarks (Fig. 1) -its most likely fate [5, 6]. Although the Higgs boson decays this way $58 \%$ of the time, the process is much more difficult to observe than some less probable decay channels.

The weak-interaction theory conceived by Weinberg and Salam was ultimately subsumed into the standard model of particle physics [7]. The Higgs mechanism is the linchpin of this theory, explaining not only the masses of the $W$ and $Z$ bosons but also providing a way to account for the masses of the fundamental fermions - the quarks and charged leptons. In the standard model, the fermions couple directly to the Higgs field via the so-called Yukawa interactions, which then generate the fermion masses and the couplings of the fermions to the Higgs boson. Alternatives to the standard model implementation of the Higgs mechanism exist, but they are less economical, requiring new layers of complexity to account for the fermion masses we see in nature [8]. Still, experimental data must be the final arbiter.

To test the Higgs mechanism as employed by the standard model, experimentalists measure the strength of the Higgs boson interactions with other fundamental particles. The strength of the Higgs-fermion interaction is proportional to the fermion mass and is therefore greatest for the top 


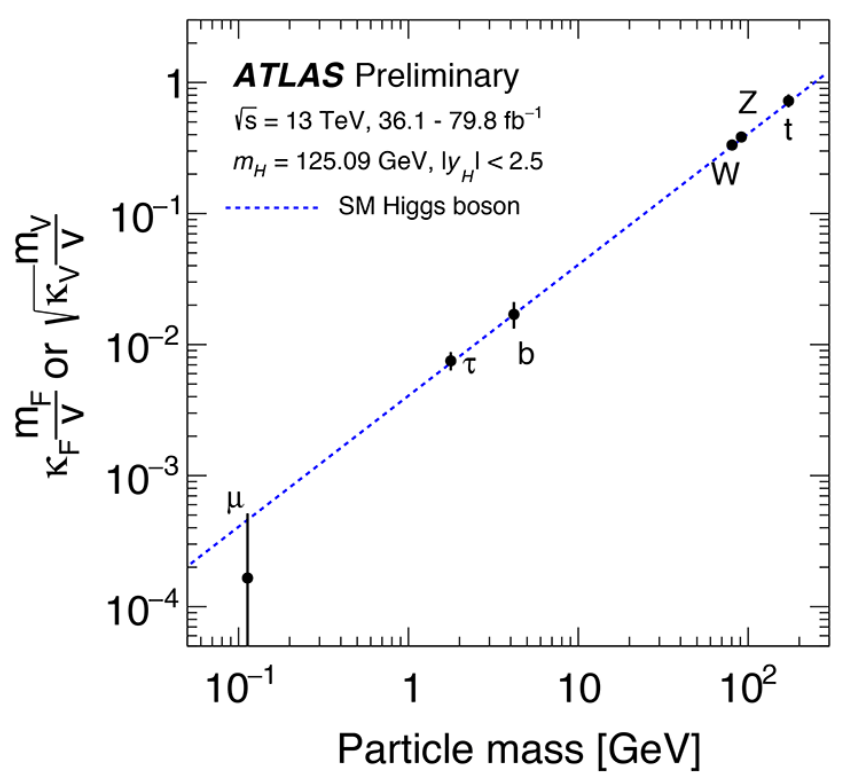

Figure 2: In the standard model, the strength of the interaction between the Higgs boson and a fundamental fermion ( $y$ axis) is proportional to the fermion mass ( $x$ axis), as indicated by the dotted blue line. The plot also shows the experimentally measured Higgs-fermion interaction strengths and the (suitably rescaled) interaction strengths between the Higgs boson and the $W$ and $Z$ bosons, respectively. (ATLAS

Collaboration/ATLAS-CONF-2018-031)

quark-the heavist quark (Fig. 2). Moreover, the stronger the interaction the larger the decay rate of the Higgs boson into a pair of the fermions, and hence the more probable the decay. Since the top quark is heavier than the Higgs boson, the most likely decay is into a pair of bottom quarks $(H \rightarrow b \bar{b})$, the heaviest decay products allowed by energy conservation.

Naively, one might have expected the discovery of the Higgs boson to have come from observing its most probable decay. But this decay mode is extremely problematic. Huge numbers of bottom-quark pairs are produced directly by proton-proton collisions, creating a background of $10^{7}$ pairs for each pair produced from a Higgs boson decay. Picking out a signal from such a huge background is nearly impossible. For this reason, the initial hunt for the Higgs boson at CERN focused on two rare decay channels with more manageable backgrounds: a decay into two photons and a decay into four charged leptons via a pair of $Z$ bosons, which occur with a probability of $2 \times 10^{-3}$ and $10^{-4}$, respectively. Observing these rare decays was sufficient to claim the discovery of the Higgs boson, but it did not provide direct evidence for the coupling of the Higgs boson to fermions as prescribed by the standard model. Subsequent experi- ments at ATLAS and CMS established this direct coupling by observing the production of the Higgs boson in association with top quarks (see 4 June 2018 Viewpoint) [9] and the decay of the Higgs boson into a pair of tau leptons [10].

To observe the dominant decay of the Higgs boson into bottom quarks, the CMS and ATLAS researchers have had to use special methods for reducing the background. Their approach relies on the fact that proton-proton collisions sometimes produce a Higgs boson in association with either a $W$ or a $Z$ boson, known as a $V H$ event. Focusing on Higgs bosons produced in this way greatly reduces the background, bringing it to a level that is still significant, but manageable. In fact, experiments at the Tevatron at Fermilab pioneered this approach, using it to find intriguing hints for the Higgs boson ahead of the 2012 discovery at the LHC [11].

In order to identify a $\mathrm{VH}$ event, the CMS and ATLAS experimentalists use the fact that the $W$ sometimes decays into a charged lepton and a neutrino and the $Z$ sometimes decays into either a pair of neutrinos or a pair of charged leptons. These secondary particles serve as "tags" for a $\mathrm{VH}$ event. The experimentalists then look for these tags in association with two bottom-quark "jets" whose combined mass is approximately equal to that of the Higgs boson. (The jets are narrow cones of hadrons that emerge from the bottom quarks produced in the collision.) The analysis is an impressive feat, requiring a thorough understanding of the many sources of systematic error as well as the development of clever techniques for distinguishing signal-like events from background-like events.

The analysis from the CMS Collaboration is based on data taken during two experimental runs, from 2010 to 2012 and from 2015 to 2017. With $V H$ events alone, CMS observed the $H \rightarrow b \bar{b}$ decay with a statistical significance of 4.8 standard deviations (sigma). But the collaboration was also able to infer the $H \rightarrow b \bar{b}$ decay from events where the Higgs boson is produced in association with other particles. Combining these results with the observed signal from $V H$ production, they observed $H \rightarrow b \bar{b}$ with a statistical significance of 5.6 sigma-surpassing the 5-sigma level that particle physicists demand for a discovery claim. The ATLAS Collaboration pursued a similar approach and achieved a comparable result, combining data from previous searches to report an observed $H \rightarrow b \bar{b}$ signal with 5.4-sigma significance.

Both collaborations also compared the number of observed $H \rightarrow b \bar{b}$ events with the standard model expectations, which defines a ratio called the signal strength. For the $V H$ process followed by $H \rightarrow b \bar{b}$, CMS researchers measured a signal strength of $1.01 \pm 0.22$, confirming the standard model prediction with an uncertainty of roughly $20 \%$. The ATLAS measurement yielded an almost identical value.

So where are particle physicists in their pursuit to understand how the Higgs boson interacts with other particles? The measured couplings of the Higgs boson to the three 
most massive fermions-the top quark, the bottom quark, and the tau lepton-are (so far) consistent with standard model predictions. But the story is far from complete given that almost no experimental information exists on the couplings of the Higgs boson to the lighter fermions. Looking further into the future, a decade of running the LHC at high luminosity could reduce the uncertainty in the $H \rightarrow b \bar{b}$ measurement by a factor of 2 to 4 . It might also improve the sensitivity to other Higgs boson decay channels, and perhaps allow for the discovery of so-far-unobserved rare Higgs decay modes. These kinds of precision Higgs boson measurements will provide critical tests of the standard model and may even reveal the first significant cracks in the theory.

This research is published in Physical Review Letters and on the arXiv.

\section{REFERENCES}

[1] S. Weinberg, "A model of leptons," Phys. Rev. Lett. 19, 1264 (1967); A. Salam, "Weak and Electromagnetic Interactions," in Elementary Particle Physics, Proceedings of the Eighth Nobel Symposium, edited by N. Svartholm (Almqvist and Wiksell, Stockholm, 1968), p. 367.

[2] P. W. Higgs, "Broken symmetries, massless particles and gauge fields," Phys. Lett. 12, 132 (1964); F. Englert and R. Brout, "Broken symmetry and the mass of gauge vector mesons," Phys. Rev. Lett. 13, 321 (1964); G. S. Guralnik, C. R. Hagen, and T. W. B. Kibble, "Global conservation laws and massless particles," Phys. Rev. Lett. 13, 585 (1964); T. W. B. Kibble, "Symmetry breaking in non-abelian gauge theories," Phys. Rev. 155, 1554 (1967).

[3] P. W. Higgs, "Broken symmetries and the masses of gauge bosons," Phys. Rev. Lett. 13, 508 (1964); P. W. Higgs, "Sponta- neous symmetry breakdown without massless bosons," Phys. Rev. 145, 1156 (1966).

[4] G. Aad et al. (ATLAS Collaboration), "Observation of a new particle in the search for the standard model Higgs boson with the ATLAS detector at the LHC," Phys. Lett. B 716, 1 (2012); S. Chatrchyan et al. (CMS Collaboration), "Observation of a new boson at a mass of $125 \mathrm{GeV}$ with the CMS experiment at the LHC," Phys. Lett. B 716, 30 (2012).

[5] A. M. Sirunyan et al. (CMS Collaboration), "Observation of Higgs boson decay to bottom quarks," Phys. Rev. Lett. 121, 121801 (2018).

[6] M. Aaboud et al. (ATLAS Collaboration), "Observation of $H \rightarrow$ $b \bar{b}$ decays and $V H$ production with the ATLAS detector," arXiv:1808.08238.

[7] M. K. Gaillard, P. D. Grannis, and F. J. Sciulli, "The standard model of particle physics," Rev. Mod. Phys. 71, S96 (1999).

[8] C. Csáki, C. Grojean, and J. Terning, "Alternatives to an elementary Higgs," Rev. Mod. Phys. 88, 045001 (2016).

[9] M. Aaboud et al. (ATLAS Collaboration), "Evidence for the associated production of the Higgs boson and a top quark pair with the ATLAS detector," Phys. Rev. D 97, 072003 (2018); A. M. Sirunyan et al. (CMS Collaboration), "Observation of $t \bar{t} H$ production,” Phys. Rev. Lett. 120, 231801 (2018).

[10] A. M. Sirunyan et al. (CMS Collaboration), "Observation of the Higgs boson decay to a pair of $\tau$ leptons with the CMS detector," Phys. Lett. B 779, 283 (2018); G. Aad et al. (ATLAS Collaboration), "Evidence for the Higgs-boson Yukawa coupling to tau leptons with the ATLAS detector," J. High Energy Phys. 1504, 117 (2015).

[11] T. Aaltonen et al. (CDF Collaboration, Do Collaboration), "Evidence for a particle produced in association with weak bosons and decaying to a bottom-antibottom quark pair in Higgs boson searches at the Tevatron," Phys. Rev. Lett. 109, 071804 (2012).

10.1103/Physics. 11.91 